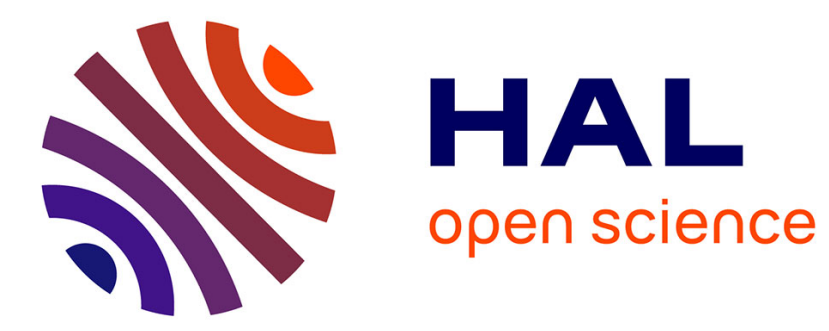

\title{
Upper bounds on the uniquely restricted chromatic index
}

\author{
Julien Baste, Dieter Rautenbach, Ignasi Sau
}

\section{To cite this version:}

Julien Baste, Dieter Rautenbach, Ignasi Sau. Upper bounds on the uniquely restricted chromatic index. Journal of Graph Theory, 2018, 91 (3), pp.251-258. 10.1002/jgt.22429 • lirmm-02412578

\section{HAL Id: lirmm-02412578 \\ https://hal-lirmm.ccsd.cnrs.fr/lirmm-02412578}

Submitted on 15 Dec 2019

HAL is a multi-disciplinary open access archive for the deposit and dissemination of scientific research documents, whether they are published or not. The documents may come from teaching and research institutions in France or abroad, or from public or private research centers.
L'archive ouverte pluridisciplinaire HAL, est destinée au dépôt et à la diffusion de documents scientifiques de niveau recherche, publiés ou non, émanant des établissements d'enseignement et de recherche français ou étrangers, des laboratoires publics ou privés. 


\title{
Upper Bounds on the Uniquely Restricted Chromatic Index
}

\author{
Julien Baste ${ }^{1} \quad$ Dieter Rautenbach ${ }^{2} \quad$ Ignasi Sau ${ }^{3}$ \\ ${ }^{1}$ CNRS, LIRMM, Université de Montpellier, Montpellier, France \\ baste@lirmm.fr, sau@lirmm.fr \\ ${ }^{2}$ Institute of Optimization and Operations Research, Ulm University, Germany \\ dieter.rautenbach@uni-ulm.de
}

\begin{abstract}
Golumbic, Hirst, and Lewenstein define a matching in a simple, finite, and undirected graph $G$ to be uniquely restricted if no other matching covers exactly the same set of vertices. We consider uniquely restricted edge-colorings of $G$ defined as partitions of its edge set into uniquely restricted matchings, and study the uniquely restricted chromatic index $\chi_{u r}^{\prime}(G)$ of $G$, defined as the minimum number of uniquely restricted matchings required for such a partition.

For every graph $G$,

$$
\chi^{\prime}(G) \leq a^{\prime}(G) \leq \chi_{u r}^{\prime}(G) \leq \chi_{s}^{\prime}(G),
$$

where $\chi^{\prime}(G)$ is the classical chromatic index, $a^{\prime}(G)$ is the acyclic chromatic index, and $\chi_{s}^{\prime}(G)$ is the strong chromatic index of $G$, respectively. While Vizing's famous theorem states that $\chi^{\prime}(G)$ is either the maximum degree $\Delta(G)$ of $G$ or $\Delta(G)+1$, two famous open conjectures due to Alon, Sudakov, and Zaks, and to Erdős and Nešetřil concern upper bounds on $a^{\prime}(G)$ and $\chi_{s}^{\prime}(G)$ in terms of $\Delta(G)$. Since $\chi_{u r}^{\prime}(G)$ is sandwiched between these two parameters, studying upper bounds in terms of $\Delta(G)$ is a natural problem.

We show that $\chi_{u r}^{\prime}(G) \leq \Delta(G)^{2}$ with equality if and only if some component of $G$ is $K_{\Delta(G), \Delta(G)}$. If $G$ is connected, bipartite, and distinct from $K_{\Delta(G), \Delta(G)}$, and $\Delta(G)$ is at least 4, then, adapting Lovász's elegant proof of Brooks' theorem, we show that $\chi_{u r}^{\prime}(G) \leq \Delta(G)^{2}-\Delta(G)$. Our proofs are constructive and yield efficient algorithms to determine the corresponding edge-colorings.
\end{abstract}

Keywords: Uniquely restricted matching; edge-coloring; chromatic index; acyclic chromatic index; strong chromatic index 


\section{Introduction}

Motivated by a problem about matrices studied by Hershkowitz and Schneider [12], Golumbic, Hirst, and Lewenstein [11] introduced the notion of a uniquely restricted matching. In the present paper we consider the edge-coloring notion derived from this type of matching and provide best possible upper bounds on the corresponding chromatic index in terms of the maximum degree.

Before we explain our results in detail and discuss related research, we collect some terminology and notation. We consider finite, simple, and undirected graphs. For a graph $G$, let $V(G)$ denote its vertex set, and let $E(G)$ denote its edge set. For a vertex $u$ of $G$, the neighborhood $N_{G}(u)$ of $u$ in $G$ is $\{v \in V(G): u v \in E(G)\}$, and the closed neighborhood $N_{G}[u]$ of $u$ in $G$ is $\{u\} \cup N_{G}(u)$. A matching in $G$ is a set of pairwise non-adjacent edges of $G$. For a matching $M$, let $V(M)$ be the set of vertices incident with an edge in $M$. A matching $M$ in $G$ is induced if the subgraph $G[V(M)]$ of $G$ induced by $V(M)$ is 1-regular. Golumbic, Hirst, and Lewenstein [11] define a matching $M$ in $G$ to be uniquely restricted if there is no matching $M^{\prime}$ in $G$ with $M^{\prime} \neq M$ and $V\left(M^{\prime}\right)=V(M)$, that is, no other matching covers exactly the same set of vertices.

Each type of matching naturally leads to an edge-coloring notion. For a graph $G$, let $\chi^{\prime}(G)$ be the chromatic index of $G$, which is the minimum number of matchings into which the edge set $E(G)$ of $G$ can be partitioned. Similarly, let the strong chromatic index $\chi_{s}^{\prime}(G)[8]$ and the uniquely restricted chromatic index $\chi_{u r}^{\prime}(G)$ of $G$ be the minimum number of induced matchings and uniquely restricted matchings into which the edge set of $G$ can be partitioned, respectively. A partition of the edges of a graph $G$ into uniquely restricted matchings is a uniquely restricted edge-coloring of $G$.

Another related notion is that of an acyclic edge-coloring, which is a partition of the edge set into matchings such that the union of every two of these matchings is a forest. The minimum number of matchings in an acyclic edge-coloring of a graph $G$ is its acyclic chromatic index $a^{\prime}(G)[1,9]$.

Clearly, every induced matching is uniquely restricted. Furthermore, it is easy to see that a matching $M$ in $G$ is uniquely restricted if and only if there is no $M$-alternating cycle in $G$, which is a cycle in $G$ that alternates between edges in $M$ and edges not in $M$. This implies that every uniquely restricted edge-coloring is also an acyclic edge-coloring. These observations imply that, for every graph $G$,

$$
\chi^{\prime}(G) \leq a^{\prime}(G) \leq \chi_{u r}^{\prime}(G) \leq \chi_{s}^{\prime}(G)
$$

Vizing's classical result [18] states that $\chi^{\prime}(G)$ of a graph $G$ of maximum degree $\Delta$ is either $\Delta$ or $\Delta+1$, and two well known open conjectures concern upper bounds on $\chi_{s}^{\prime}(G)$ and $a^{\prime}(G)$ in terms of $\Delta$. Erdös and Nešetřil (see [8]) conjectured $\chi_{s}^{\prime}(G) \leq \frac{5}{4} \Delta^{2}$, and much of the research on the strong chromatic index is motivated by this conjecture. Building on earlier work of Molloy and Reed [17], and Bruhn and Joos [4], Bonamy, Perrett, and Postle $[3]$ showed $\chi_{s}^{\prime}(G) \leq 1.835 \Delta^{2}$ provided that $\Delta$ is sufficiently large. For further results on the strong chromatic index we refer to $[2,8,13,14]$. Fiamčik [9] and Alon, Sudakov, and Zaks [1] conjectured $a^{\prime}(G) \leq \Delta+2$. See $[5,6,10]$ for further references and the currently best known results concerning general graphs and graphs of large girth. In view of these open conjectures, the inequality chain (1) motivates to study upper bounds on $\chi_{u r}^{\prime}(G)$ in terms of the maximum degree $\Delta$ of a graph $G$. Our contribution are best-possible such bounds and the characterization of all extremal graphs. Since our proofs are constructive, it is easy to extract efficient algorithms finding the corresponding edge-colorings. 


\section{Upper bounds on $\chi_{u r}^{\prime}(G)$}

Our first result applies to general graphs, and its proof relies on a natural greedy strategy. Faudree, Schelp, Gyárfás, and Tuza [7] conjectured $\chi_{s}^{\prime}(G) \leq \Delta^{2}$ for a bipartite graph $G$ of maximum degree $\Delta$, and our Theorem 2.3 can be considered to be a weak version of this conjecture. Theorem 2.3 below shows that excluding the unique extremal graph from Theorem 2.1, the uniquely restricted chromatic index of bipartite graphs drops considerably.

For an integer $k$, let $[k]$ be the set of all positive integers at most $k$.

Theorem 2.1. If $G$ is a connected graph of maximum degree at most $\Delta$, then $\chi_{u r}^{\prime}(G) \leq \Delta^{2}$ with equality if and only if $G$ is $K_{\Delta, \Delta}$.

Proof. Since no two edges of $K_{\Delta, \Delta}$ form a uniquely restricted matching in this graph, we obtain $\chi_{u r}^{\prime}\left(K_{\Delta, \Delta}\right)=$ $\left|E\left(K_{\Delta, \Delta}\right)\right|=\Delta^{2}$. Now, let $G$ be a connected graph of maximum degree at most $\Delta$. We first show that $\chi_{u r}^{\prime}(G) \leq \Delta^{2}$. In a second step, we show that $\chi_{u r}^{\prime}(G)<\Delta^{2}$ provided that $G$ is not $K_{\Delta, \Delta}$.

We consider the vertices of $G$ in some linear order, say $u_{1}, \ldots, u_{n}$. For $i$ from 1 up to $n$, we assume that the edges of $G$ incident with vertices in $\left\{u_{1}, \ldots, u_{i-1}\right\}$ have already been colored, and we color all edges between $u_{i}$ and $\left\{u_{i+1}, \ldots, u_{n}\right\}$ using distinct colors, and avoiding any color that has already been used on a previously colored edge incident with some neighbor of $u_{i}$. Since $u_{i}$ has at most $\Delta$ neighbors, each of which is incident with at most $\Delta$ edges, this procedure requires at most $\Delta^{2}$ many distinct colors.

Suppose, for a contradiction, that some color class $M$ is not a uniquely restricted matching in $G$. Since $M$ is a matching by construction, there is an $M$-alternating cycle $C$. Let $C: u_{r_{1}} u_{s_{1}} u_{r_{2}} u_{s_{2}} \ldots u_{r_{k}} u_{s_{k}} u_{r_{1}}$ be such that $r_{1}$ is the minimum index of any vertex on $C$, and $u_{r_{1}} u_{s_{k}} \in M$. These choices trivially imply $r_{1}<s_{1}$ and $r_{1}<r_{2}$. If $r_{2}>s_{1}$, then $u_{r_{1}} u_{s_{k}} \in M$ implies that, when coloring the edge $u_{s_{1}} u_{r_{2}}$, some edge incident with the neighbor $u_{r_{1}}$ of $u_{s_{1}}$ would already have been assigned the color of the edges in $M$, and the above procedure would have avoided this color on $u_{s_{1}} u_{r_{2}}$. Therefore, since $u_{r_{1}} u_{s_{k}} \in M$ and $u_{r_{2}} u_{s_{1}} \in M$, the coloring rules imply $r_{2}<s_{1}$, that is, $r_{1}<r_{2}<s_{1}$. Now, suppose that $r_{i}<r_{i+1}<s_{i}$ for some $i \in[k-1]$. Since $u_{r_{i+1}} u_{s_{i}} \in M$ and $u_{r_{i+2}} u_{s_{i+1}} \in M$, the coloring rules imply in turn

- $r_{i+1}<s_{i+1}$, since otherwise we would have colored $u_{r_{i+1}} u_{s_{i}}$ differently,

- $r_{i+2}<s_{i+1}$, since otherwise we would have colored $u_{r_{i+2}} u_{s_{i+1}}$ differently, and

- $r_{i+1}<r_{i+2}$, since otherwise we would have colored $u_{r_{i+1}} u_{s_{i}}$ differently.

It follows that $r_{i+1}<r_{i+2}<s_{i+1}$, where we identify $r_{k+1}$ with $r_{1}$. Now, by an inductive argument, we obtain $r_{1}<r_{2}<\cdots<r_{k}<r_{1}$, which is a contradiction.

Altogether, we obtain $\chi_{u r}^{\prime}(G) \leq \Delta^{2}$.

Now, let $G$ be distinct from $K_{\Delta, \Delta}$, and we want to prove that $\chi_{u r}^{\prime}(G)<\Delta^{2}$. Among all uniquely restricted edge-colorings of $G$ using colors in $\left[\Delta^{2}\right]$, we choose a coloring for which the number of edges with color 1 is as small as possible. Clearly, we may assume that some edge $u v$ has color 1 , as otherwise we already have that $\chi_{u r}^{\prime}(G)<\Delta^{2}$.

If there is a color $\alpha$ in $\left[\Delta^{2}\right] \backslash\{1\}$ such that no edge incident with a neighbor of $u$ has color $\alpha$, then changing the color of $u v$ to $\alpha$ yields a uniquely restricted edge-coloring of $G$ with less edges of color 1 , 
which is a contradiction. In view of the maximum degree, this implies that every vertex in $N_{G}[u]$ has degree $\Delta$, the set $N_{G}(u)$ is independent, and, for every color $\alpha$ in $\left[\Delta^{2}\right]$, there is exactly one edge incident with a neighbor of $u$ that has color $\alpha$.

Since $G$ is not $K_{\Delta, \Delta}$, some neighbor $x$ of $u$ has a neighbor $y$ that does not lie in $N_{G}(v)$. Without loss of generality, let $u x$ have color 2 , and let $x y$ have color 3 . Let $M$ be the set of edges with color 3 .

If $G$ does not contain an $M$-alternating path of odd length (number of edges) at least 3 between $x$ and a vertex in $N_{G}(v) \backslash\{u\}$ that contains the edge $x y$, then changing the color of $u v$ to 3 yields a uniquely restricted edge-coloring of $G$ with less edges of color 1, which is a contradiction. Hence, $G$ contains such a path, which implies that two edges incident with neighbors of $y$ have color 3 .

If there is a color $\alpha$ in $\left[\Delta^{2}\right] \backslash\{1\}$ such that no edge incident with a neighbor of $y$ has color $\alpha$, then changing the color of $x y$ to $\alpha$ and the color of $u v$ to 3 yields a uniquely restricted edge-coloring of $G$ with less edges of color 1 , which is a contradiction. Similarly as above, this implies that, for every color $\alpha$ in $\left[\Delta^{2}\right] \backslash\{1,3\}$, there is exactly one edge incident with a neighbor of $y$ that has color $\alpha$. Now, changing the color of $u v$ to 2 , the color of $u x$ to 3 , and the color of $x y$ to 2 yields a uniquely restricted edge-coloring of $G$ with less edges of color 1 , which is a contradiction. This completes the proof.

As observed above, the proof of Theorem 2.1 is algorithmic; the simple greedy strategy considered in its first half efficiently constructs uniquely restricted edge-colorings using at most $\Delta^{2}$ colors. Furthermore, also its second half can be turned into an efficient algorithm that finds uniquely restricted edge-colorings using at most $\Delta^{2}-1$ colors for connected graphs of maximum degree $\Delta$ that are distinct from $K_{\Delta, \Delta}$; the different cases considered in the proof correspond to simple manipulations of a given uniquely restricted edge-coloring that iteratively reduce the number of edges of color 1 down to 0. Golumbic, Hirst, and Lewenstein [11] showed that deciding whether a given matching is uniquely restricted can be done in polynomial time, and their algorithm can be used to decide which of the simple manipulations can be executed.

Our next goal is to improve Theorem 2.1 for bipartite graphs. The following proof was inspired by Lovász's [16] elegant proof of Brooks' Theorem.

Lemma 2.2. If $G$ is a connected bipartite graph of maximum degree at most $\Delta \geq 4$ that is distinct from $K_{\Delta, \Delta}$, and $M$ is a matching in $G$, then $M$ can be partitioned into at most $\Delta-1$ uniquely restricted matchings in $G$.

Proof. Let $A$ and $B$ be the partite sets of $G$, and let $R=V(G) \backslash V(M)$. Note that $M$ is perfect if and only if $R$ is empty. Whenever we consider a coloring of the edges in $M$, and $\alpha$ is one of the colors, let $M_{\alpha}$ be the set of edges in $M$ colored with $\alpha$.

First, we assume that $R$ is empty, and that $G$ is not $\Delta$-regular. By symmetry, we may assume that some vertex $a$ in $A$ has degree less than $\Delta$. Let $a b \in M$. Let $T$ be a spanning tree of $G$ that contains the edges in $M$. Contracting within $T$ the edges from $M$, rooting the resulting tree at the vertex corresponding to the edge $a b$, and considering a breadth-first search order, we obtain the existence of a linear order $a_{1} b_{1}, \ldots, a_{n} b_{n}$ of the edges in $M$ such that $a b=a_{n} b_{n}$, and, for every $i \in[n-1]$, there is an edge between $\left\{a_{i}, b_{i}\right\}$ and $\left\{a_{i+1}, b_{i+1}, \ldots, a_{n}, b_{n}\right\}$. Since $a_{n}$ has degree less than $\Delta$, this implies that, for every $i \in[n]$, some vertex $u_{i}$ in $\left\{a_{i}, b_{i}\right\}$ has at most $\Delta-2$ neighbors in $\left\{a_{1}, b_{1}, \ldots, a_{i-1}, b_{i-1}\right\}$. Now, we color the edges in $M$ greedily in the above linear order. Specifically, for every $i$ from 1 up to $n$, we color 
the edge $a_{i} b_{i}$ with some color $\alpha$ in $[\Delta-1]$ such that, for every $j \in[i-1]$, for which $u_{i} \in\left\{a_{i}, b_{i}\right\}$ has a neighbor in $\left\{a_{j}, b_{j}\right\}$, the edge $a_{j} b_{j}$ is not colored with $\alpha$. By the degree condition on $u_{i}$, such a coloring exists. Suppose, for a contradiction, that $M_{\alpha}$ is not uniquely restricted for some color $\alpha$ in $[\Delta-1]$. Let the edge $a_{i} b_{i}$ in $M_{\alpha}$ be such that it belongs to some $M_{\alpha}$-alternating cycle $C$, and, subject to this condition, the index $i$ is maximum. If the neighbor of $u_{i}$ on $C$ outside of $\left\{a_{i}, b_{i}\right\}$ is in $\left\{a_{j}, b_{j}\right\}$, then the choice of the edge $a_{i} b_{i}$ implies $j<i$, and the coloring rule implies that the edge $a_{j} b_{j}$ is not colored with $\alpha$, which is a contradiction. Altogether, the statement follows.

Next, we assume that $R$ is non-empty. Let $K$ be a component of $G-R$. Let $M_{K}$ be the set of edges in $M$ that lie in $K$. Since $G$ is connected, the graph $K$ is not $\Delta$-regular. Therefore, proceeding exactly as above, we obtain a coloring of the edges in $M_{K}$ using the colors in [ $\left.\Delta-1\right]$ such that each color class is a uniquely restricted matching in $K$. If $K_{1}, \ldots, K_{k}$ are the components of $G-R$, and $M_{i}$ is a uniquely restricted matching in $K_{i}$ for every $i \in[k]$, then $M_{1} \cup \cdots \cup M_{k}$ is a uniquely restricted matching in $G$. Therefore, combining the colorings within the different components, we obtain that also in this case the statement follows.

At this point, we may assume that $G$ is $\Delta$-regular, and that $M$ is perfect.

Next, we assume that there are two distinct edges $e$ and $e^{\prime}$ in $M$ such that $V\left(\left\{e, e^{\prime}\right\}\right)$ is a vertex cut of $G$. This implies that we can partition the set $M \backslash\left\{e, e^{\prime}\right\}$ into two non-empty sets $M_{1}$ and $M_{2}$ such that there is no edge between $V\left(M_{1}\right)$ and $V\left(M_{2}\right)$. For $i \in[2]$, let $G_{i}$ be the subgraph of $G$ induced by $V\left(\left\{e, e^{\prime}\right\} \cup M_{i}\right)$. Since $G$ is connected, the graph $G_{i}$ is not $\Delta$-regular. In view of the above, this implies that there is a coloring $c_{i}$ of the edges of the perfect matching $\left\{e, e^{\prime}\right\} \cup M_{i}$ of $G_{i}$ using the colors in $[\Delta-1]$ such that each color class of $c_{i}$ is a uniquely restricted matching in $G_{i}$. If $c_{i}(e) \neq c_{i}\left(e^{\prime}\right)$ for both $i$ in [2], then we may assume that $c_{1}$ and $c_{2}$ assign the same colors to $e$ and $e^{\prime}$, and it is easy to verify that the common extension $c$ of $c_{1}$ and $c_{2}$ to $M$ has the property that every color class of $c$ is a uniquely restricted matching in $G$. Hence, we may assume that necessarily $c_{1}(e)=c_{1}\left(e^{\prime}\right)$. Note that this implies in particular that at least one of the two possible edges between $V(\{e\})$ and $V\left(\left\{e^{\prime}\right\}\right)$ is missing.

Let $c_{1}(e)=\alpha$. Let $e=a b, e^{\prime}=a^{\prime} b^{\prime}$, and $U=\left\{a, b, a^{\prime}, b^{\prime}\right\}$. For every vertex $u \in U$, let $C_{1}(u)$ be the set of colors $\beta$ for which $M_{1}$ contains an edge $v w$ with $c_{1}(v w)=\beta$ such that $u$ is adjacent to $v$ or $w$. If there is some $u \in U$ and some color $\beta \in([\Delta-1] \backslash\{\alpha\}) \backslash C_{1}(u)$, then changing the color of the unique edge in $\left\{e, e^{\prime}\right\}$ incident with $u$ from $\alpha$ to $\beta$ yields a coloring $c_{1}^{\prime}$ of the edges in $\left\{e, e^{\prime}\right\} \cup M_{1}$ using the colors in $[\Delta-1]$ such that each color class of $c_{1}^{\prime}$ is a uniquely restricted matching in $G_{1}$. Furthermore, $c_{1}^{\prime}(e) \neq c_{1}^{\prime}\left(e^{\prime}\right)$, which is a contradiction. This implies that $[\Delta-1] \backslash\{\alpha\} \subseteq C_{1}(u)$ for every $u \in U$. In particular, each vertex $u$ in $U$ has at least $\Delta-2$ neighbors in $V\left(M_{1}\right)$, and, hence, at most one neighbor in $V\left(M_{2}\right)$. Let $C_{2}(u)$ for $u \in U$ be defined analogously as above. Clearly, the set $C_{2}(a) \cup C_{2}\left(a^{\prime}\right)$ contains at most two distinct colors. Since $\Delta-1 \geq 3$, we may assume that $c_{2}$ is such that the set $C_{2}(a) \cup C_{2}\left(a^{\prime}\right)$ does not contain the color $\alpha$. Now, let $c_{2}^{\prime}$ be a coloring of the edges in $\left\{e, e^{\prime}\right\} \cup M_{2}$ that coincides with $c_{2}$ on $M_{2}$ and colors $e$ and $e^{\prime}$ with color $\alpha$. It is easy to see that each color class of $c_{2}^{\prime}$ is a uniquely restricted matching in $G_{2}$. Let $c$ be the common extension of $c_{1}$ and $c_{2}^{\prime}$ to $M$. Suppose, for a contradiction, that the color class $M_{\beta}$ of $c$ is not uniquely restricted for some color $\beta$ in $[\Delta-1]$. Clearly, we have $\beta=\alpha$. Let $C$ be an $M_{\alpha}$-alternating cycle in $G$. It is easy to see that $C$ contains both edges $e$ and $e^{\prime}$, but no edge between $\{a, b\}$ and $\left\{a^{\prime}, b^{\prime}\right\}$. Furthermore, it follows that $C$ contains an edge between $\left\{a, a^{\prime}\right\}$ and $V\left(M_{2}\right)$. Since $c$ coincides with $c_{2}$ on $M_{2}$, and $C_{2}(a) \cup C_{2}\left(a^{\prime}\right)$ does not contain $\alpha$, we obtain a contradiction. 
Altogether, we may assume that there are no two distinct edges $e$ and $e^{\prime}$ in $M$ such that $V\left(\left\{e, e^{\prime}\right\}\right)$ is a vertex cut of $G$.

Now, we show the existence of three edges $a b, a^{\prime} b^{\prime}$, and $a^{\prime \prime} b^{\prime \prime}$ in $M$ such that some of the two possible edges between $\left\{a^{\prime}, b^{\prime}\right\}$ and $\left\{a^{\prime \prime}, b^{\prime \prime}\right\}$ is missing, and either $a$ is adjacent to $b^{\prime}$ as well as $b^{\prime \prime}$ or $b$ is adjacent to $a^{\prime}$ as well as $a^{\prime \prime}$. Therefore, let $a_{1} b_{1}$ be an edge in $M$. Let $a_{2} b_{2}, \ldots, a_{\Delta} b_{\Delta}$ be the edges in $M$ such that $N_{G}\left(a_{1}\right)=\left\{b_{1}, \ldots, b_{\Delta}\right\}$. We may assume that $\left\{a_{2}, b_{2}, \ldots, a_{\Delta}, b_{\Delta}\right\}$ induces a complete bipartite graph $K_{\Delta-1, \Delta-1}$; otherwise, we find the three edges with the desired properties. Since $G$ is not $K_{\Delta, \Delta}$, the vertex $b_{1}$ is non-adjacent to some vertex $a_{i}$ in $\left\{a_{2}, \ldots, a_{\Delta}\right\}$. Now, if $a_{j} \in\left\{a_{2}, \ldots, a_{\Delta}\right\} \backslash\left\{a_{i}\right\}$, then one of the two possible edges between $\left\{a_{1}, b_{1}\right\}$ and $\left\{a_{i}, b_{i}\right\}$ is missing, and $b_{j}$ is adjacent to $a_{1}$ as well as $a_{i}$. Altogether, we obtain three edges $a b, a^{\prime} b^{\prime}$, and $a^{\prime \prime} b^{\prime \prime}$ in $M$ with the desired properties.

By symmetry, we may assume that $a$ is adjacent to $b^{\prime}$ and $b^{\prime \prime}$, and $a^{\prime}$ is non-adjacent to $b^{\prime \prime}$. In view of the above, the graph $G^{\prime}=G-V\left(\left\{a^{\prime} b^{\prime}, a^{\prime \prime} b^{\prime \prime}\right\}\right)$ is connected, and $M^{\prime}=M \backslash\left\{a^{\prime} b^{\prime}, a^{\prime \prime} b^{\prime \prime}\right\}$ is a perfect matching of $G^{\prime}$. Let $T^{\prime}$ be a spanning tree of $G^{\prime}$ that contains the edges in $M^{\prime}$. Contracting within $T^{\prime}$ the edges from $M^{\prime}$, rooting the resulting tree in the vertex corresponding to the edge $a b$, and considering a breadth-first search order, we obtain the existence of a linear order $a_{3} b_{3}, \ldots, a_{n} b_{n}$ of the edges in $M^{\prime}$ such that $a b=a_{n} b_{n}$, and, for every $i \in[n-1] \backslash[2]$, there is an edge between $\left\{a_{i}, b_{i}\right\}$ and $\left\{a_{i+1}, b_{i+1}, \ldots, a_{n}, b_{n}\right\}$. Now, we color the edges in $M$ greedily in the linear order $a_{1} b_{1}, a_{2} b_{2}, a_{3} b_{3}, \ldots, a_{n} b_{n}$, where $a_{1} b_{1}=a^{\prime \prime} b^{\prime \prime}$ and $a_{2} b_{2}=a^{\prime} b^{\prime}$. Note that, for every $i \in[n-1] \backslash[2]$, some vertex $u_{i}$ in $\left\{a_{i}, b_{i}\right\}$ has at most $\Delta-2$ neighbors in $\left\{a_{1}, b_{1}, \ldots, a_{i-1}, b_{i-1}\right\}$. We color $a_{1} b_{1}$ and $a_{2} b_{2}$ with the same color. For every $i$ from 3 up to $n-1$, we color the edge $a_{i} b_{i}$ with a color $\alpha$ in $[\Delta-1]$ such that, for every $j \in[i-1]$, for which $u_{i}$ has a neighbor in $\left\{a_{j}, b_{j}\right\}$, the edge $a_{j} b_{j}$ is not colored with $\alpha$. By the degree condition on $u_{i}$, such a coloring exists. Finally, since $a_{n}$ has neighbors in the two edges $a_{1} b_{1}$ and $a_{2} b_{2}$ that are colored with the same color, there is some color $\alpha$ in $[\Delta-1]$ for which no edge $a_{i} b_{i}$ with $i \in[n-1]$ such that $a_{n}$ is adjacent to $b_{i}$, is colored with $\alpha$, and we color the edge $a_{n} b_{n}$ with that color $\alpha$. Suppose, for a contradiction, that $M_{\beta}$ is not uniquely restricted for some color $\beta$ in $[\Delta-1]$. Let the edge $a_{i} b_{i}$ in $M_{\beta}$ be such that it belongs to some $M_{\beta}$-alternating cycle $C$, and, subject to this condition, the index $i$ is maximum. Since $a^{\prime}$ is non-adjacent to $b^{\prime \prime}$, we have $i \geq 3$. Let $u_{n}=a_{n}$. If the neighbor of $u_{i}$ on $C$ outside of $\left\{a_{i}, b_{i}\right\}$ is in $\left\{a_{j}, b_{j}\right\}$, then the choice of the edge $a_{i} b_{i}$ implies $j<i$, and the coloring rule implies that the edge $a_{j} b_{j}$ is not colored with $\beta$, which is a contradiction. This completes the proof.

Lemma 2.2 fails for $\Delta=3$; the matching $\left\{a_{1} b_{1}, a_{2} b_{2}, a_{3} b_{3}, a_{4} b_{4}, a_{5} b_{5}\right\}$ of the graph $G$ in Figure 1 cannot be partitioned into two uniquely restricted matchings. Note that the matching $\left\{a_{1} b_{3}, a_{2} b_{1}, a_{3} b_{5}, a_{4} b_{2}, a_{5} b_{4}\right\}$ though is the union of the two uniquely restricted matchings $\left\{a_{1} b_{3}, a_{3} b_{5}\right\}$ and $\left\{a_{2} b_{1}, a_{4} b_{2}, a_{5} b_{4}\right\}$.

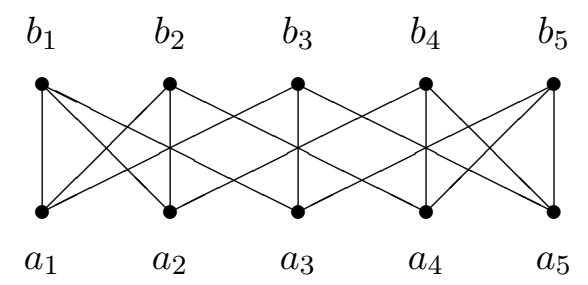

Figure 1: A bipartite graph $G$.

Lemma 2.2 also fails for non-bipartite graphs; in fact, if $G$ arises from the disjoint union of two copies 
of $K_{\Delta}$ by adding a perfect matching $M$, then every partition of $M$ into uniquely restricted matchings requires $\Delta$ sets.

With Lemma 2.2 at hand, the proof of our final result is easy.

Theorem 2.3. If $G$ is a connected bipartite graph of maximum degree at most $\Delta \geq 4$ that is distinct from $K_{\Delta, \Delta}$, then $\chi_{u r}^{\prime}(G) \leq \Delta^{2}-\Delta$.

Proof. Since $G$ is bipartite, its edge set can be partitioned into $\Delta$ matchings [15]. By Lemma 2.2, each of these matchings can be partitioned into $\Delta-1$ uniquely restricted matchings. This completes the proof.

Note that the graph $G$ in Figure 1 also satisfies $\chi_{u r}^{\prime}(G) \leq \Delta^{2}-\Delta=9-3=6$. In fact, the uniquely restricted matchings $\left\{a_{1} b_{1}, a_{4} b_{2}, a_{5} b_{4}\right\},\left\{a_{1} b_{2}, a_{2} b_{4}, a_{5} b_{5}\right\},\left\{a_{2} b_{1}, a_{3} b_{3}, a_{4} b_{5}\right\},\left\{a_{1} b_{3}, a_{4} b_{4}\right\},\left\{a_{2} b_{2}, a_{3} b_{5}\right\}$, and $\left\{a_{3} b_{1}, a_{5} b_{3}\right\}$ partition $E(G)$.

\section{References}

[1] N. Alon, B. Sudakov, and A. Zaks, Acyclic edge colorings of graphs, Journal of Graph Theory 37 (2001) 157-167.

[2] L.D. Andersen, The strong chromatic index of a cubic graph is at most 10, Discrete Mathematics 108 (1992) 231-252.

[3] M. Bonamy, T. Perrett, and L. Postle, Colouring Graphs with Sparse Neighbourhoods: Bounds and Applications, submitted.

[4] H. Bruhn and F. Joos, A stronger bound for the strong chromatic index, Combinatorics, Probability and Computing 27 (2018) 21-43.

[5] X.S. Cai, G. Perarnau, B. Reed, and A.B. Watts, Acyclic edge colourings of graphs with large girth, Random Structures and Algorithms 50 (2017) 511-533.

[6] L. Esperet and A. Parreau, Acyclic edge-coloring using entropy compression, European Journal of Combinatorics 34 (2013) 1019-1027.

[7] R.J. Faudree, A. Gyárfás, R.H. Schelp, and Zs. Tuza, Induced matchings in bipartite graphs, Discrete Mathematics 78 (1989) 83-87.

[8] R.J. Faudree, R.H. Schelp, A. Gyárfás, and Zs. Tuza, The strong chromatic index of graphs, Ars Combinatoria 29B (1990) 205-211.

[9] J. Fiamčik, The acyclic chromatic class of a graph, Mathematica Slovaca 28 (1978) 139-145.

[10] I. Giotis, L. Kirousis, K.I. Psaromiligkos, D.M. Thilikos, Acyclic edge coloring through the Lovász Local Lemma, Theoretical Computer Science 665 (2017) 40-50.

[11] M.C. Golumbic, T. Hirst, and M. Lewenstein, Uniquely restricted matchings, Algorithmica 31 (2001) 139-154. 
[12] D. Hershkowitz and H. Schneider, Ranks of zero patterns and sign patterns, Linear Multilinear Algebra 34 (1993) 3-19.

[13] H. Hocquard, M. Montassier, A. Raspaud, and P. Valicov, On strong edge-colouring of subcubic graphs, Discrete Applied Mathematics 161 (2013) 2467-2479.

[14] H. Hocquard, P. Ochem, and P. Valicov, Strong edge-colouring and induced matchings, Information Processing Letters 113 (2013) 836-843.

[15] D. Kőnig, Graphen und Matrizen, Matematikai és Fizikai Lapok 38 (1931), 116-119.

[16] L. Lovász, Three short proofs in graph theory, Journal of Combinatorial Theory, Series B 19 (1975) 269-271.

[17] M. Molloy and B. Reed, A bound on the strong chromatic index of a graph, Journal of Combinatorial Theory, Series B 69 (1997) 103-109.

[18] V.G. Vizing, On an estimate of the chromatic class of a p-graph, Diskretnyj Analiz 3 (1964) 25-30. 